\title{
Some aspects of architectural lighting of historical buildings
}

\author{
M. Górczewska \\ Poznan University of Technology, \\ Institute of Electrical and Electronics Engineering, Poland
}

\begin{abstract}
The aim of night illumination is to recall the whole object image, as it is in the daylight city panorama. In building illumination we reach for solutions which are suitable for object function and architecture. Illumination with light cast from different directions is to expose details of the object's architecture by play of light and shadow on facades and details of the building. Architectural lighting is also often used inside. Architectural interior lighting uses a number of formal solutions, in which light is emitted from behind the edges screening the luminaires. This kind of solution gives indirect illumination of ceilings or walls and light sources are invisible.

This article discusses the role, tasks of illuminating, and architectural lighting and presents lighting effects obtained for design implementation, where the architecture and indoor arrangement or the building's façade architecture were linked with architectural lighting.

Keywords: lighting engineering, architectural lighting.
\end{abstract}

\section{Introduction}

The modern lighting techniques, applied for creating the monuments nocturnal appearance, should include aspects concerning the architecture as well as symbolic meaning of the illuminated facilities or buildings. The realization of these principles is connected with the experience and imagination of the designer as well as with the technical possibilities, i.e. mainly with the parameters of applied light sources and luminaires. 


\section{Indoor illumination}

The illumination is particularly important in the interiors of the commonly accessible historical facilities or buildings, particularly when they become the location for various cultural, artistic and special events. In the mentioned interiors the lighting, apart from its usable functions, is also used for illumination functions, when by the illumination there are considered actions with use of the lighting for the purpose of creating atmosphere, exhibiting with use of the light elements and details of the architecture and creating in the interior space the specific sight guide leading through its more important elements. It is necessary to look for such solutions that could bring the required lighting effect without disturbing too much these interiors' character $[1,2]$.

The proper lighting inside interiors of the historical buildings or facilities, should meet requirements put by the usable function, physiology of seeing process and need of the appropriate exhibiting the symbolic, historical and esthetic values of illuminated interiors.

The particular challenge for the lighting realization, are the interiors of the sacral facilities or buildings. In the sacral space, there are taking place the direct interactions between symbols, liturgy and architecture and for that reason every design of the lighting should take into consideration these church elements. The light in the church has got a significant influence on the church functioning, its perception by the participants and visitors. The artificial lighting should imitate, if possible, the natural lighting. It should also help the participants in concentrating their attention on the liturgical ceremony actions and even possibly underline them. It is necessary to remember that the lighting should help with perception and not disturb.

During performing the liturgical ceremony, the vertical illuminance should be dominating for the purpose of concentrating the attention of the participants on the ceremony course. The lighting should be installed in such a way to create a kind of the light hierarchy where the altar becomes the most important.

The light should provide comfort to the participants as well as the possibility to admire the architecture and decorative interior of the church. The lighting system should also be designed to include conditions influencing the operation and function of the church.

Until now, inside the sacral facilities interiors, there have been used mainly the chandeliers and wall luminaires with standard incandescent light sources. Such lighting has been very often insufficient and provided limited possibilities to adopt the light quantity to needs.

However, sill more often there are applied the modern lighting systems. Within these systems, there are used new energy-saving and durable light sources as well as the appropriate luminaires. The historical chandeliers or wall luminaires can become the decorative elements only for the purpose of underlying the interior character.

The need of creating the atmosphere of concentration or self-communion with distributing at the same time the lighting accents appropriately to the needs of the liturgical ceremony and occasional events (such as wedding ceremonies), causes 
that the good solution consists in applying a number of luminaires having the appropriate technical and photometric parameters. These luminaires should be installed in the way that possibly harmonize with the architecture of the church interior or with the architecture of the fragment of the interior, without disturbing the interior esthetic of such specific facilities or buildings.

Obtaining the appropriate lighting effects without exhibiting luminaires is possible with use of the light sources characterized by high lighting efficiency with relatively small dimensions.

In order to illuminate the chancel and the altar for liturgical purposes, projectors with metal halide sources with the intensity of 20 to $150 \mathrm{~W}$ giving warm light might be very useful. To illuminate the architectural details, sculptures, polychromy etc. low-voltage halogen sources with the power of 20 to 100W are often used, with properly distributed luminous intensity. Ceilings, cornices, niches, etc. architectural details may be displayed using linear sources of light, i.e. by various types of fluorescent or LED lamps. Big advantage of such sources of light is their diversity, durability and high luminous efficiency as well as the possibility to adjust the luminous flux.

An example of such realization is the lighting system applied in the church of Virgin Mary Sorrowful in Poznan - fig. 1.
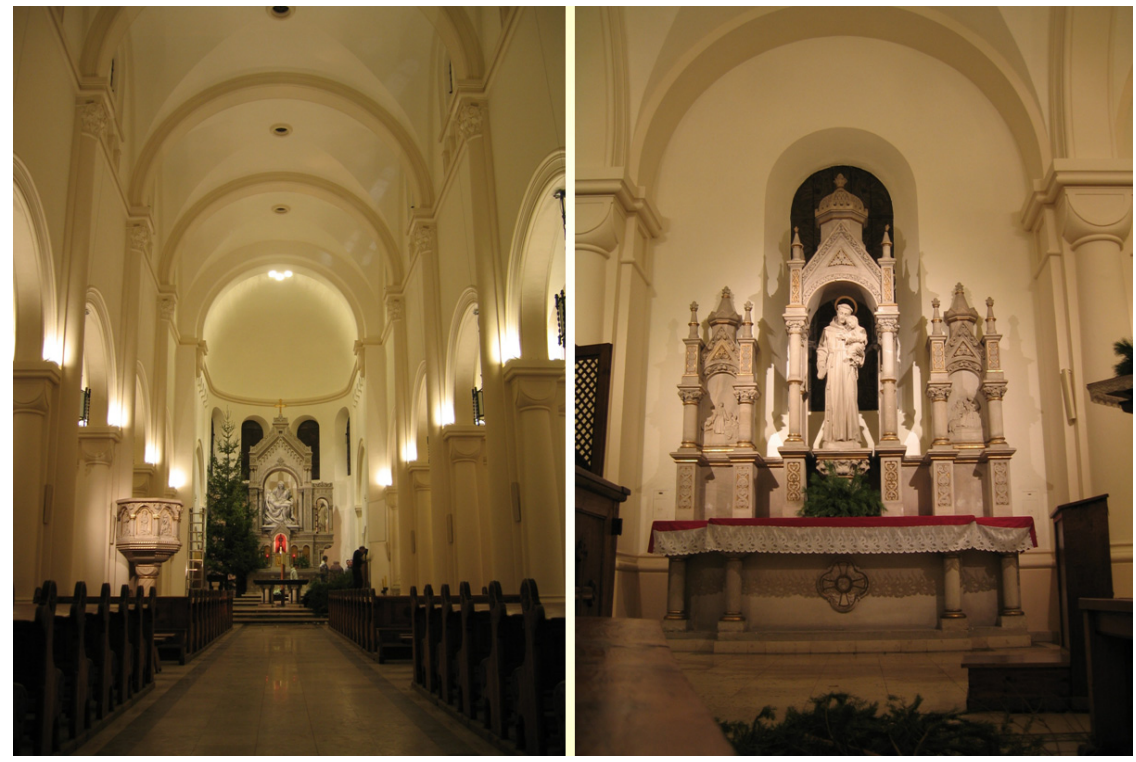

Figure 1: Interior of the church of Virgin Mary Sorrowful.

In the lighting system of this interior, there have been used the traditional solutions - i.e. hanging lamps with applied modern fluorescent lamps as well as flood-lights fitted in the ceiling and located over cornices the specially fabricated luminaires equipped with the compact fluorescent lamps and spot halogen lamps. 


\section{Illumination of the historical buildings}

The monumental buildings illumination is a particular task. The facilities or buildings outline viewed in a daily light is less distinct, even in case of facilities or buildings of rich architecture, because the daily light produces flood lighting that in practice illuminates elevations in the uniform way.

The nocturnal illumination with use of the light arriving from different directions, allows completely different configuration of the building body and elevation elements, i.e. windows, portals, columns, attics, towers, domes, etc. The illumination is an occasion to create with use of the light the requested atmosphere and produce or create with use of the light the symbolic meaning of the facility or building. The atmosphere can be formed by the lighting dynamics and light color.

The lighting dynamics can express itself in the illuminance diversification on the elevation elements and in creating lighting plans. It can be achieved by repeating the light spots and shadows adopted to the rhythm of the elevation architectonic elements. Operating the light plans gives the illumination lighting depth through showing for example outlines of finials on the background of the walls illuminated in the further plan.

The light color in the illumination co-create the atmosphere together with the light dynamics. The light color should harmonize with the elevation coloring or color of the facing material used to cover architectonical elements.

The illumination becomes also the factor that informs about meaning and significance of the illuminated facility or building. The view of non illuminated facilities of buildings very often becomes the cause of memorizing the negative impression. This impression results also when the elevation luminance of the illuminated facility or building is too low when comparing with the high luminance of the street lighting. Therefore it is so important to analyze the facility or building surrounding.

The elaboration of the illumination project is connected with the necessity of evaluating a number of conditions which influence the finally accepted technical solutions.

Generally speaking, such issue includes the evaluation of:

- $\quad$ esthetical and emotional aspects,

- technical and economic aspects.

The esthetical and emotional criteria are mainly related with the analysis of site prospective of the facilities or buildings, their historical value and importance, architecture attractiveness, etc.

The technical and economic criteria are related with the choice of the appropriate light sources and luminaires, location and assembling, power and control system.

Over a half of monuments in Poland are sacral facilities or buildings. Their role and importance in the history caused that churches and monasteries became the real treasury of the most valuable art pieces and monuments of the material culture history and moreover the architecture and interior decorations became the regional history record of the regional society and the nation as a whole. There 
are many cities where the church is the only monumental building. The importance of this fact results in the installed illuminations.

The modern lighting techniques, utilized to create the nocturnal appearance of the monuments and monumental buildings, should include aspects related with the architecture as well as with symbolic representation of the illuminated facilities or buildings.

The realization of these principles is strictly conditioned by the technical possibilities, i.e. the implemented illumination method and parameters of the applied light sources and luminaires.

In the illumination designing practice, there is applied the flood-lighting method and spot-lighting method or both of them mixed together at the same time. Each of the methods is creating different conditions for the realization.

Until not long ago, the most of the facilities or buildings has been illuminated with use of the flood-lighting method by using for this purpose a small number of the high power luminaires. This method has been found appropriate for illuminating the facilities or building of huge dimensions, viewed form a large distance. The example of such illumination is the illumination of the Cathedral in Gniezno (Poland).

The Gniezno Archicathedral Basilica is a sacred building of historic importance and a monument of national culture. Its history is closely connected with the birth of the Polish state and Christianity. It was created as a Roman structure, then it was rebuilt in Gothic style, which is displayed in many details of the Cathedral architecture. Baroque also influenced the shape and particularly the decoration of the Cathedral chapels.

The Gniezno Cathedral is a high structure situated on a natural elevation Lech Hill, which makes the towers and the roof of the nave visible from different directions and from quite a long distance. The characteristic Cathedral towers silhouetted against the view of Gniezno remind us of the values which this church fosters.

The Cathedral illumination project was developed after making an analysis of visibility of particular facades. It appeared that the front facade and both side facades are seen mainly from a greater distance. Whereas the facades of the presbytery apse are seen in the frame of the street leading from the Cathedral to the Gniezno Market.

This had a crucial impact on the illumination method chosen, because from a greater distance the structure is perceived mostly as a set of solids - the towers, nave and aisles. At close range watchers are impressed by architectural details figures, bas-reliefs, stained-glass windows, etc. The desired illumination effect has been attained by selecting appropriate levels of illumination of particular parts of the Cathedral, as well as by diversifying light color. In general about 40 projectors with a total power of $15 \mathrm{~kW}$ have been used. Flood-lights with halogen, sodium and metal-halide sources with daylight, white and warm light color have been used.

It should be stressed that the electrical system has been designed to enable both cost-effective illumination and the desired visual effect.

The illuminated Cathedral from the main vista side is seen in fig. 2. 


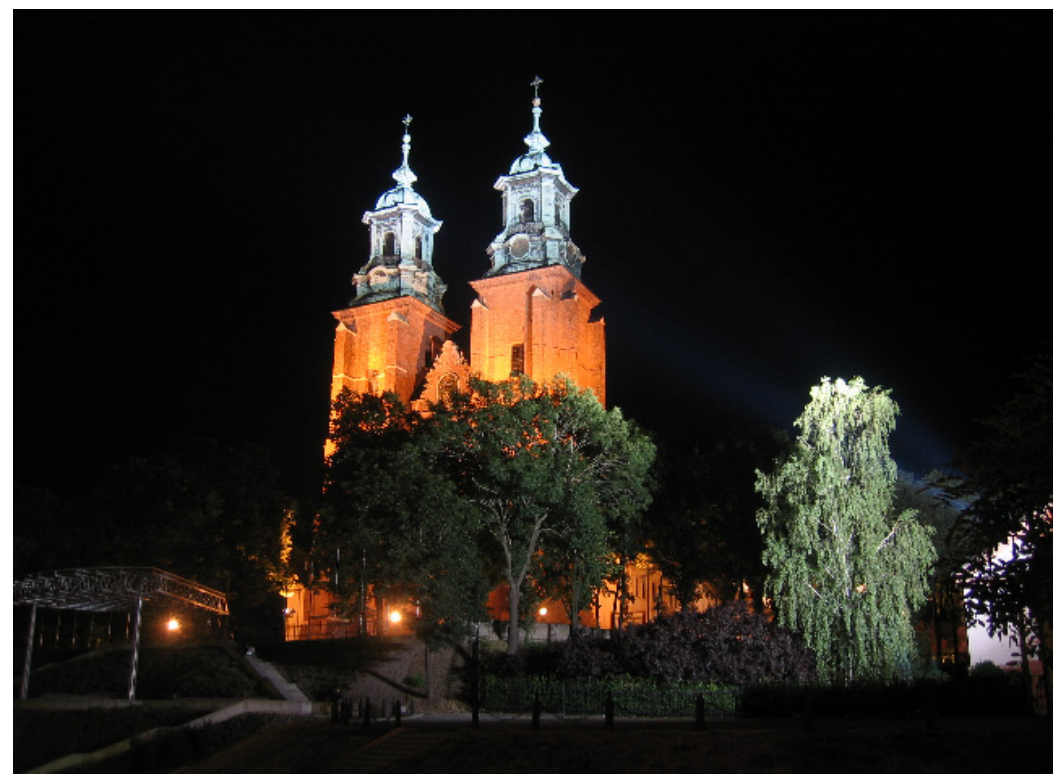

Figure 2: $\quad$ Illumination of Cathedral in Gniezno.

The external illumination project has been accompanied by a change in the internal lighting. The existing candelabrums with incandescent lamps have been replaced with fittings with discharge light sources (white soda and metal-halide) - fig. 3.

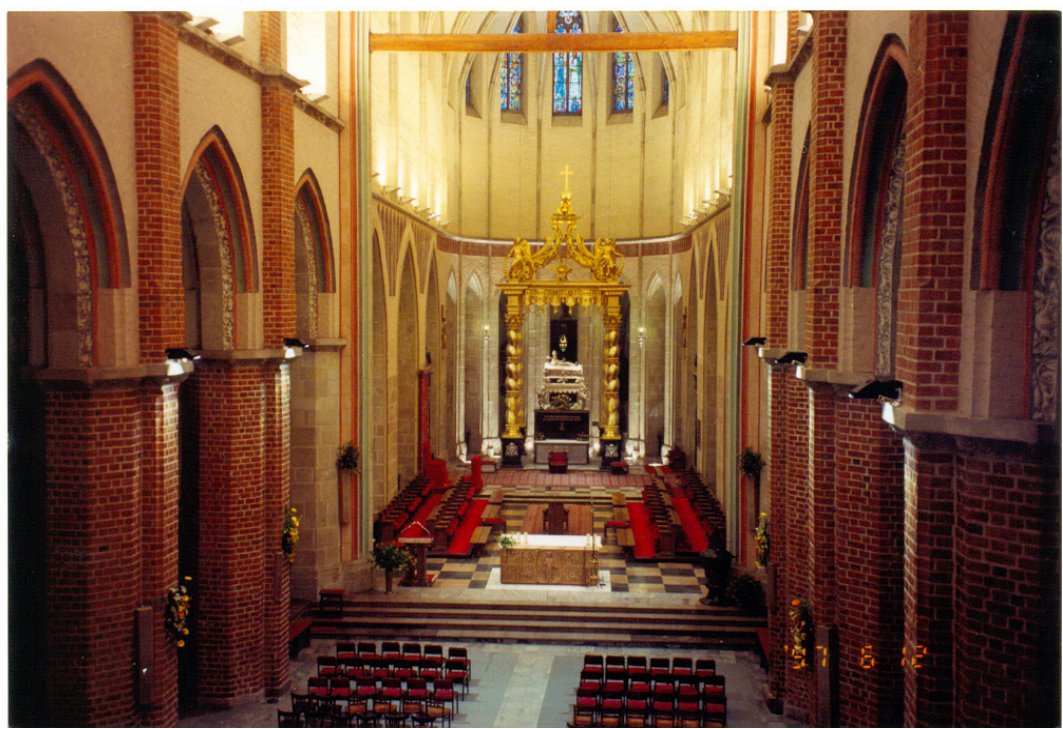

Figure 3: $\quad$ Interior of the Cathedral. 
For the facilities or buildings of small dimensions and limited site-seeing or viewing prospective, the artistic effect of the realized in such a way illumination not always meets expectations, in particular when referring to the facilities or buildings viewed form a short distance.

Within the last years, the spot-lighting method of the illumination becomes more frequently applied, with use of a bigger number of the low power luminaires. This type of the illumination allows more sufficiently underlying the significant architecture details and moreover allows concentrating the viewers' attention on details which very often skip the attention in the daylight.

The small dimension light sources give the possibility of obtaining in the luminaires good optical conditions and allow assembling the luminaires directly on the buildings elevations. This method of the illumination realization provides more flexibility in operating the light and shadow proportions.

An example of such realization is the illumination of the Collegium Maius in Poznań - fig. 4.
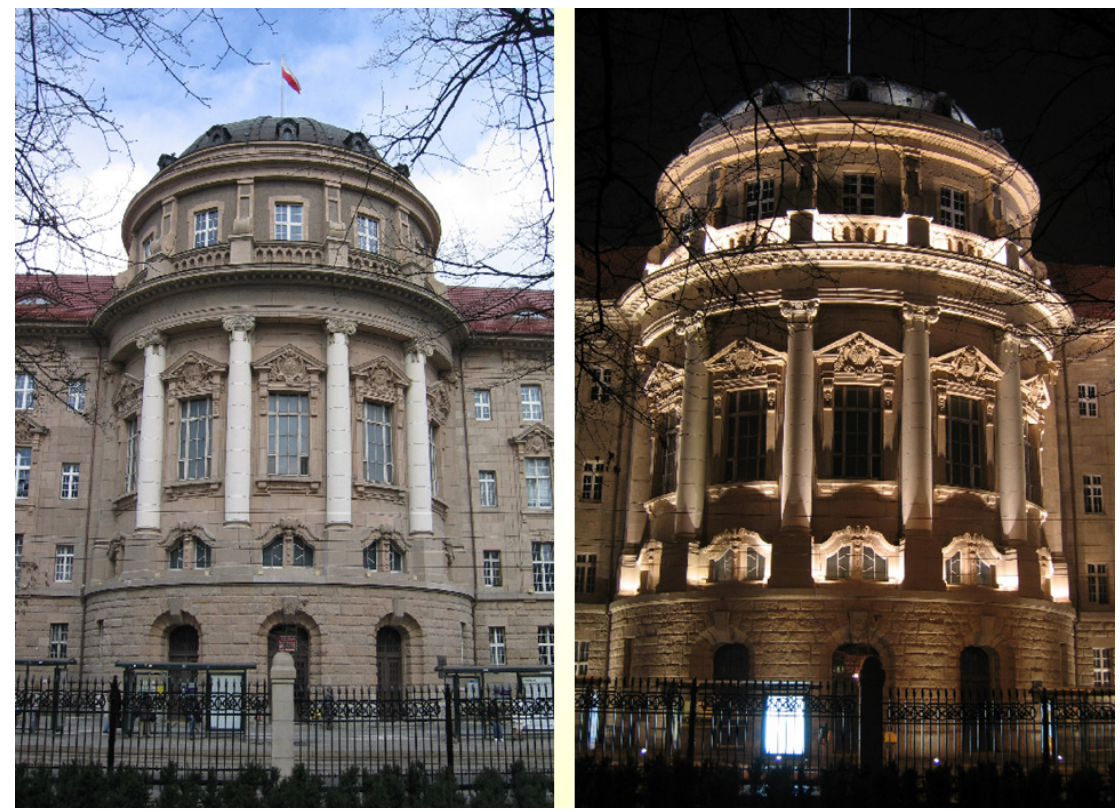

Figure 4: $\quad$ Illumination of the front side of Collegium Maius.

The photographs show some examples of illumination effect of front side and of individual fragments of the Collegium Maius building. The light that tangently illuminates the façades and their architectural details emphasized the color and the texture of the stone and the abundance of decorative forms. This way, the authentic character of the building has been featured. The lighting effects achieved will make a new, attractive image of the University Building.

The illumination of the Collegium Maius needed in total 122 luminaires with the capacity from $35 \mathrm{~W}$ to $150 \mathrm{~W}$. 
Applying the spot-lighting method in the illumination with using the luminaires assembled on the building elevation, has got certain limitations. Very often the historians of art and conservators of monuments are against this type of the illumination. In their opinion, illuminating the architectonic details from below creates strong shadows above horizontal details - which is opposite to the natural structure of shadows and excessively exhibits the vertical system of the elevation.

So, there results a problem to be solved: how to illuminate the facility or building viewed form a short distance prospective and at the same time reconcile the need of creating the attractive appearance with conservation limits concerning the way of illuminating the architectonical details as well as the method and location of the luminaires assembling.

Distribution of the photosensitive elements of the human eye retina is the reason that the reception of the surrounding us reality relies upon synthesis of the detailed impressions originated from the small, central field of view and upon general impressions originated from the wider peripheral field of view.

Within the vision process of the nature, we watch it not in a continuous and uniform way in every point but by leaps with individual looks which are attracted by interesting details. These places are directed the biggest numbers of looks.

When utilizing this feature of the vision process, it is possible to construct the attractive nocturnal appearance of the illuminated facility or building by the appropriate distribution of the lighting accents on the elevation and creating the specific illumination guide for the vision of the viewer.

The realization of the illumination lighting with utilizing the principle of creating the nocturnal appearance of the facility or building by positioning on its surface the light spots of different luminance represents the compromise between too monotonous flood-lighting and spot-lighting creating diversified and very often too theatrical appearance of the illuminated facility or building.

This way of illuminating simplifies adopting to the conservation requirements and limits and at the same time allows maintaining the possibility of obtaining the required esthetical and emotional effect. For obtaining this purpose it is convenient to apply:

- diversified light sources having relatively small power,

- luminaires of the appropriately selected and directed light distribution.

This way of the realization of the illumination does not require assembling the luminaires on the facility or building. The example of such illumination is the illumination of the church of St. Joseph and Bernardine church in Poznań.

The St. Joseph church, located on St. Wojciech Hill, is the example of the early baroque architecture. The decorative front elevation is characterized by a certain rhythm of pilasters divided by recesses. The system of cornices divides the elevation into three levels. The church is surrounded by high trees and so it is very well visible mainly from the front.

For the illumination lighting of the church front, there have been used the luminaires equipped with the sodium light sources "white soda" type 100W power, assembled in the ground as well as on the ground - fig. 5. The obtained illumination result has been presented on fig. 6 . 


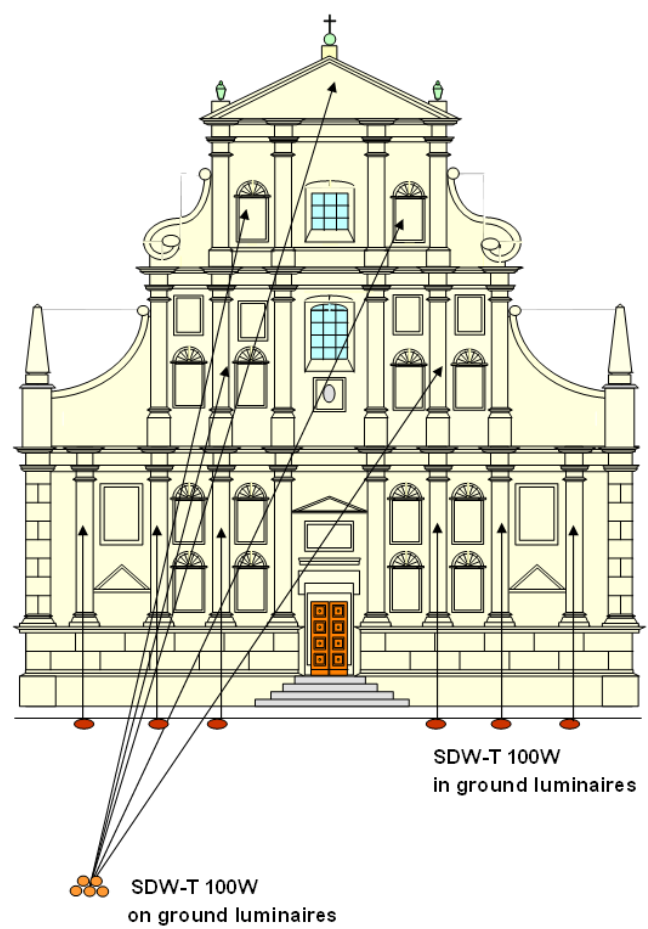

Figure 5: Front elevation of St. Joseph church- the position of luminaires.
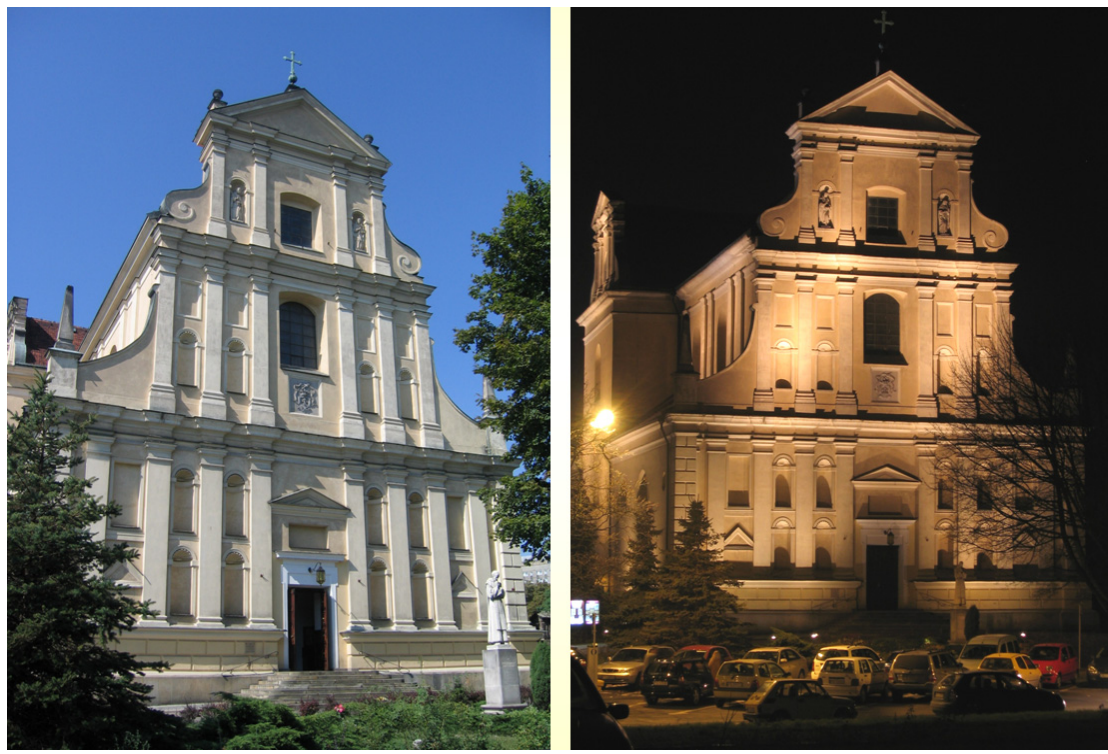

Figure 6: Front elevation of St. Joseph church - daily and nocturnal view. 
The church of St. Francisco from Assisi (or Bernardine church) is the example of the baroque architecture. The front elevation is characterized by the rhythm of pilasters divided by recesses. The system of cornices divides the elevation into three levels. The elevations are covered with plaster. Roofs and helmets of high towers are covered with the copper glazed sheet.

The church and in particular its towers are well visible from the more distant prospective. From the shorter prospective of Bernardine Square only the front elevation is visible.

For the illumination lighting of the church front and towers, there have been applied the luminaires equipped with the metal halogen light sources of power from 35 up to $150 \mathrm{~W}$, assembled on the existing street lighting pillars. The obtained illumination result has been presented on fig. 7 .
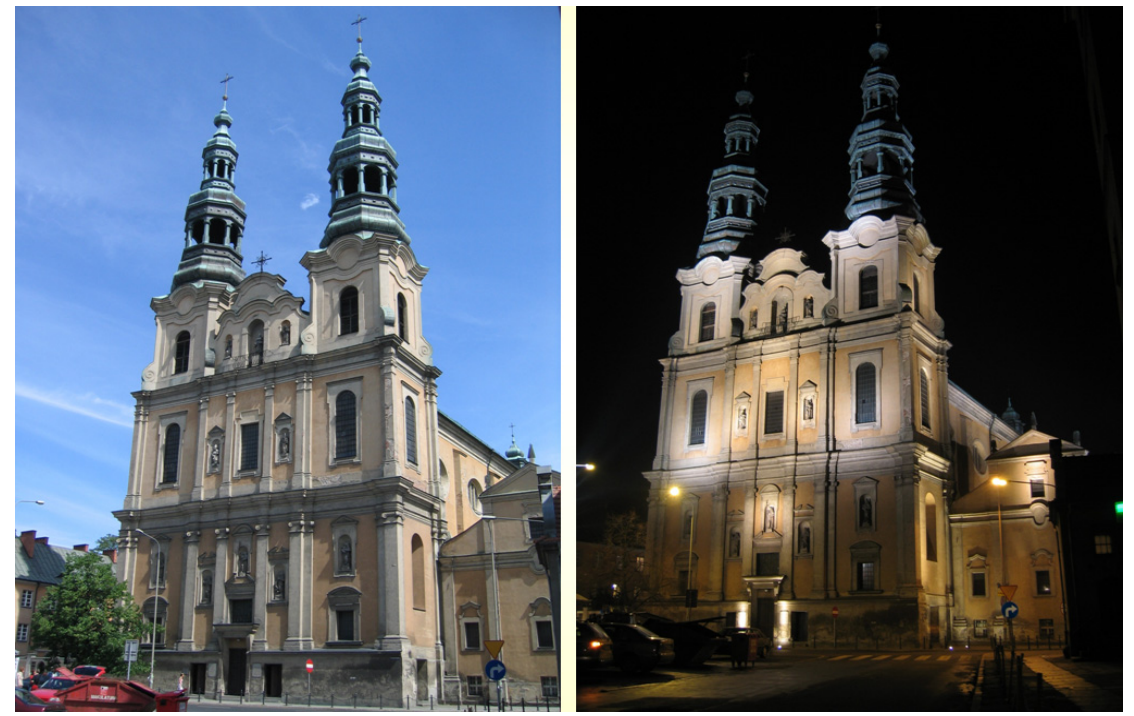

Figure 7: Front elevation of St. Francisco church - daily and nocturnal view.

\section{Conclusions}

The usable lighting is insufficient for creating the appropriate atmosphere and exhibiting the interior decorative elements as well as architecture of the buildings. And it is why there is a need of applying additional, individually designed lighting systems which become the important and valuable element for creating the attractive appearance of the facilities and buildings during the night.

\section{References}

[1] Żagan, W., Iluminacja obiektów, Oficyna Wyd. Politechniki Warszawskiej, Warszawa, 2003.

[2] CIE Technical Report No 94 - Guide for Floodlighting. 